\author{
Berend DENKENA ${ }^{1}$ \\ Benjamin BERGMANN ${ }^{1}$ \\ Miriam HANDRUP ${ }^{*}$ \\ Matthias WITT ${ }^{1}$
}

\title{
MATERIAL IDENTIFICATION DURING TURNING BY NEURAL NETWORK
}

\begin{abstract}
A design concept for high-performance components involves the combination of different materials in hybrid workpieces. Different material properties and chemical compositions influence the machining quality of hybrid workpieces. To achieve a constant workpiece and process quality, it is necessary to adjust the process parameters to the individual material. Thus, it is mandatory to classify the material during machining for the relevant range of process parameters. This paper examines teaching strategies for neural networks to determine the machined material in process by a small amount of cross points. For this purpose, different training sets are compared. Process parameters with different cutting speeds, feeds and with constant and varying depth of cut are examined. In addition, the signal sources necessary for robust material classification are compared and investigated. The investigation is performed for the cylindrical turning of friction welded EN AW-6082/20MnCr5 shafts. The study shows that an F1 score of 0.99 is achieved at a constant cutting depth, provided that only the corner points of the process window and the machine control signals are used for training. With an additional variation of the cutting depth, the classification rate is significantly improved by the use of external sensors such as the acceleration sensor.
\end{abstract}

\section{INTRODUCTION}

Reducing the weight of components is one of the most effective ways to decrease $\mathrm{CO}_{2}$ emissions and fuel consumption in the automotive and aircraft industries [1]. A lightweight construction strategy for highly loaded components is to combine different materials in one part to adapt the material properties to the occurring load by creating workpiece compounds [2]. After joining the materials, a forming process is carried out to improve the micro-structure in the joining zone [3-5]. After forming, a cutting process is performed to adjust the geometry, surface roughness and shape. Cutting characteristics and chip formation mechanisms change during machining based on different material properties and chemical compositions and can lead to poor surface quality and geometrical errors. Boehnke described the occurring effects for a turning process of workpiece compounds [6]. He studied the influence of process parameters and cutting tool geometry on the shape of the compounds. In addition, he indicated which actions are required for a process design based on workpiece quality. One aspect is

\footnotetext{
${ }^{1}$ Institute of Production Engineering and Machine Tools (IFW), Leibniz Universität Hannover, Germany

*Email: handrup@ifw.uni-hannover.de https://doi.org/10.36897/jme/119677
} 
the customization of the tool design. Ozsváth investigated the optimization of the milling process for magnesium-steel joints by adjusting the tool geometry [7]. The cutting force, surface roughness, chip formation and chip temperature served as the basis for evaluation. He concluded that even with optimized geometry of the indexable insert tip, both materials have different surface roughnesses if no additional parameter adjustment is made. Denkena et al. showed that a material-specific adaptation of the process parameters offers the potential to improve the process quality in terms of surface quality, cutting tool load, machining time and residual stresses [8]. To machine hybrid workpieces material-specifically, the position of the material transition must be known. However, this varies due to production fluctuations in the previous subprocesses. For this reason, it is necessary to classify which material is currently being processed to guarantee a material-specific adaptation of the process parameters. This requires information from the process, which is obtained by machine data and external sensors. The sensor data can be fused to obtain a monitoring with high robustness and sensitivity. Machine learning methods serve as a possible approach to classify the materials based on the different signals.

Neural networks (NN) provide a framework for classifying process states during processing by learning from previously recorded data. In addition, they use parallel calculations suitable for real-time monitoring and can also be used to extract complex trends and solve nonlinear problems [9]. For this reason, they are used in various fields of process monitoring [10-12]. Denkena et al. investigated various machine learning approaches for the classification of hybrid material compounds [13]. They indicated the suitability of an NN to separate materials with significantly different material properties such as aluminum and steel. The investigations were performed for a fixed set of feed, cutting speed and cutting depth. However, since the process parameters can change depending on the objective such as surface quality or material removal rate in the process, it is necessary to consider different process parameters. To minimize the effort for the training of the $\mathrm{NN}$ as much as possible, an efficient teaching strategy must be researched to train the NN with as little data as possible. This includes investigating how robust an NN that has been trained for relevant feeds and cutting speeds behaves to a variation of the cutting depth. Furthermore, the robustness of classifycation and its dependency on the selection of the signal process have not yet been investigated.

Therefore, this paper focuses for the first time on the transferability of the NN for material classification to relevant process parameters to increase flexibility in the machining of hybrid components. Section 3 examines the classification rate for different teaching strategies that consider different sets of parameters in order to reduce the effort required to train the NN. Section 4 investigates which signal sources must be minimally available for robust material identification based on NN. Signals from the machine control, acceleration sensors and process forces measured with a dynamometer are compared.

\section{METHODOLOGY}

Experimental tests were carried out on the turning center Gildemeister CTX420 linear. An industrial personal computer (IPC) was connected to an open platform communication 
server and fieldbus interface of the Siemens Powerline 840D machine control. For the cylindrical turning, an indexable insert DNMG150404-FP5 by Walter AG was used. The material properties of the friction welded hybrid shaft consisting of an aluminum-steel compound EN AW-6082/20MnCr5 are presented in Table 1.

Table 1. Material properties

\begin{tabular}{|c|c|c|}
\cline { 2 - 3 } \multicolumn{1}{c|}{} & $20 \mathrm{MnCr} 5$ & EN AW-6082 \\
\hline Density $\left[\mathrm{g} / \mathrm{cm}^{3}\right]$ & 7.75 & 2.70 \\
\hline Hardness $[\mathrm{HB}]$ & 255 & 95 \\
\hline Ultimate tensile strength $[\mathrm{MPa}]$ & $980-1280$ & 310 \\
\hline Young's modulus $[\mathrm{GPa}]$ & 210 & 70 \\
\hline Thermal expansion coefficient $\left[10^{-6} \mathrm{~K}^{-1}\right]$ & 11.5 & 23.4 \\
\hline Conductivity $[\mathrm{W} / \mathrm{mK}]$ & 42 & $170-220$ \\
\hline
\end{tabular}

The properties of the machined material, which are directly linked to the process characteristics and force progression, affect the chip formation. These include plasticity, structural strength and flow stresses. Thus, the process force and vibration during machining differ if the material properties have larger deviations. To measure the vibration during the process, two acceleration sensors were mounted on the tool turret $a_{\text {turret }}$ and tool $a_{\text {tool. }}$. The sampling rate for both sensors was $10000 \mathrm{~Hz}$. In addition, a dynamometer was used to measure process forces recorded at a sampling rate of $1000 \mathrm{~Hz}$. The passive force $F_{p}$ was orientated in $x$-direction, the cutting force $F_{c}$ in $y$-direction and the feed force $F_{f}$ in $z$-direction. The spindle torque $M_{\text {spindle }}$ and the motor current for each axis direction $I_{X, Y, Z}$ are provided by the Numerical Control (NC) at $83 \mathrm{~Hz}$, as depicted in Fig. 1a.

Initially, the cutting depth was set constant at $a_{p}=1 \mathrm{~mm}$. The cutting speed $v_{c}$ was changed in three steps from $200 \mathrm{~m} / \mathrm{min}$ to $400 \mathrm{~m} / \mathrm{min}$ and the feed $f$ was varied in three steps from $0.1 \mathrm{~mm}$ to $0.4 \mathrm{~mm}$, as depicted in Fig. 1b. The aim of this investigation was to see how the NN performs in a range of relevant process parameters. Afterwards, the process window was extended into the third dimension by additionally considering the cutting depth. For this purpose, $a_{p}$ was varied by $\pm 0.5 \mathrm{~mm}$ and $v_{c}$ as well as $f$ were placed between the previously considered process parameter sets, as depicted in Fig. 1b. These extended investigations served to validate the robustness of the NN against process variations such as geometrical fluctuations. Based on the measured signals, training sets for the machine learning algorithm were determined. For signal processing, the offsets of the signals were subtracted and the acceleration signals were filtered with a high-pass filter. Afterwards, various features were generated from each signal. The mean value $\bar{x}$, root mean square (RMS), standard deviation $\sigma$, peak-to-rms (P2R) and peak-to-peak (P2P) were calculated for each signal with a running window sized $0.1 \mathrm{~s}$. The features were then labelled with the knowledge of the material sequence in the process and the material boundaries.

The consideration of variables with low information value can significantly affect the performance of the classifier. Therefore, the most sensitive features are identified before application. 

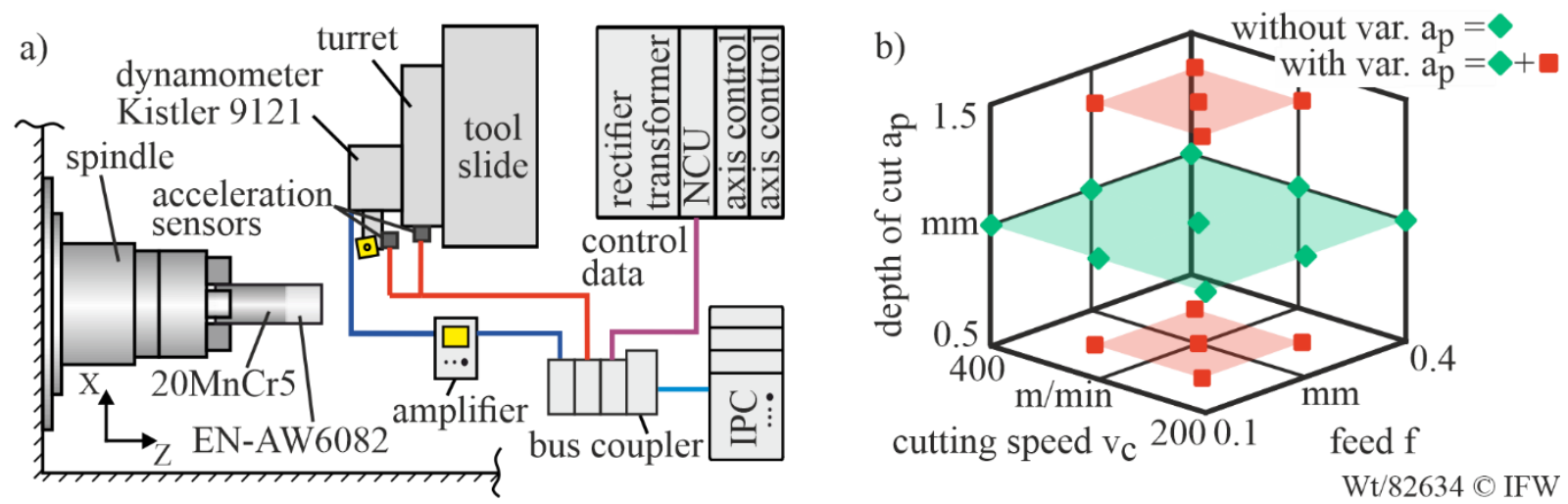

Fig. 1. Measuring setup and signal path (a), investigated process parameter sets (b)

One method of selecting the best characteristics is to calculate the statistical overlap factor $(S O F)$. The $S O F$ determines the degree of separation of a feature between the first and second material. The correlation between the features is not taken into account by the $S O F$. The $S O F$ favours features with a high degree of separation and low variance due to different material properties.

$$
S O F=\left|\frac{\bar{x}_{1}-\bar{x}_{2}}{\sigma_{1}+\sigma_{2}}\right|
$$

Of all available features, the ten highest rated features were transferred into the training set, presented in Table 2. This number provides a compromise between the information content and the required computing effort. As the $\mathrm{NN}$ is to be used for different process parameter sets, the parameters $v_{c}$ and $f$ were also determined by the $\mathrm{NC}$ and provided as additional input variables. The scaled conjugate gradient backpropagation was used as training algorithm. The NN used for classification was a feedforward network with 20 hidden layers. The training signals were divided randomly into training data $(70 \%)$, validation data $(15 \%)$ and test data (15\%) for the training function of the NN. Furthermore, the features were normalized to the range $[-1,1]$. For the selection of the number of layers, the number from which the accuracy of the classification converges was examined. To adjust the class distribution of the measurements, the data sets were balanced using the Synthetic Minority Oversampling Technique (SMOTE). Both materials were provided with a proportion of $40 \%$ while, the air cut represented $20 \%$ of the training data.

For the performance evaluation of the examined training strategies, the $F_{1}$ score is used. The $F_{1}$ score represents the harmonic mean of the precision and recall. While precision is defined by the percentage of relevant results, recall is defined by the percentage of total relevant results correctly classified by the algorithm. For a perfect precision and recall, the $F_{1}$ score has a value of 1 and in the worst case a value of 0 is reached. These indicators are determined based on the true positive $t p$, false positive $f p$ and false negative $f n$ of the classifying result:

$$
\text { precision }=\frac{t p}{t p+f p}
$$




$$
\begin{gathered}
\text { recall }=\frac{t p}{t p+f n} \\
F_{1}=2 \frac{\text { precision } \cdot \text { recall }}{\text { precision }+ \text { recall }}
\end{gathered}
$$

\section{MATERIAL CLASSIFICATION FOR EXTENDED PROCESS WINDOW}

First, the material identification was examined for process parameter sets with varying cutting speed $v_{c}$ and the feed $f$, while using a constant depth of cut $a_{p}$. This is used to investigate the NN's performance when the process parameter is extended from a fixed parameter set to a range of relevant process parameters. In addition, the cutting depth is varied to verify the NN's performance with process disturbances such as geometric fluctuations. Process parameter sets with a constant cutting depth of $a_{p}=1 \mathrm{~mm}$ were first examined. The four different teaching strategies which are investigated are depicted in Fig. 2a-d. Each of the measured process parameter sets is sorted either into the training set or the test set. The training set 2 a represents the teaching strategy used in the previous work [13]. Here, the $\mathrm{NN}$ is trained by a constant feed and cutting speed placed centrally in the process windows. In teaching strategies $2 b-d$, the number and position of the process parameters used for the training set are varied. While in $2 b$ the process parameters are selected so that each feed and each cutting speed is represented once, in 2c only the process parameter sets in the corner points of the process window are used for the training. Strategy $2 \mathrm{~d}$ is a combination of $2 \mathrm{a}$ and $2 \mathrm{c}$. Based on each of the presented teaching strategies, an NN is generated and the classification rate is compared.

a)

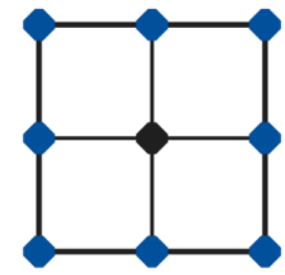

$200 \mathrm{~m} / \mathrm{min} 400$

training set b)

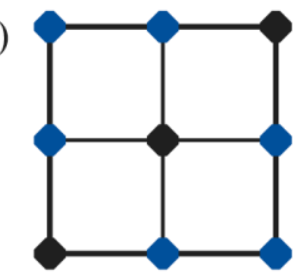

200

$\mathrm{m} / \mathrm{min} 400$ c)

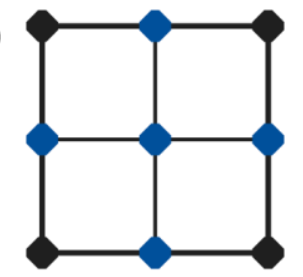

cutting speed $\mathrm{v}_{\mathrm{C}}$ d)

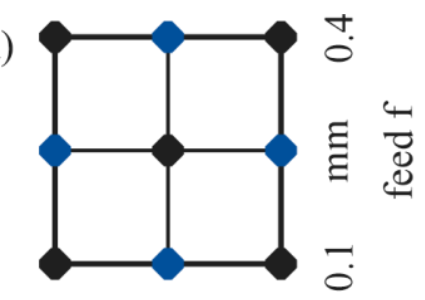

$200 \mathrm{~m} / \mathrm{min} 400$

Fig. 2. Teaching strategies for process parameter sets changing $f$ and $v_{c}$ with constant $a_{p}=1 \mathrm{~mm}$

The resulting confusion matrices in Fig. 3 show the classification rate and $F_{1}$ score of material classification for the different strategies. The materials are therefore assigned to a class: class 0 represents the air cut, class 1 the material EN AW-6082 and class 2 the material $20 \mathrm{MnCr} 5$. The NN trained with strategy 3 a only reaches an $F_{1}$ score of 0.490 . This shows that based on a single position inside the process window, it is not possible to cover a larger range. 
Only the air cut is detected. However, the NN is not able to classify the different materials for the process parameter sets arranged around them. Based on strategy $3 \mathrm{~b}$, in which three points are used for training, an $F_{1}$ score of 0.996 can already be achieved. Strategy $3 \mathrm{c}$, which uses points that frame the investigated process window, achieves an $F_{1}$ score of 0.999 . The addition of a central training point in strategy $3 \mathrm{~d}$ does not lead to a significant improvement of the classification rate.

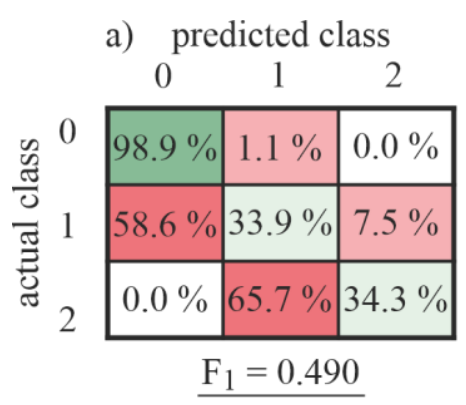

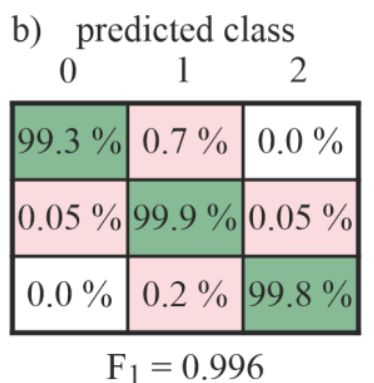

$\mathrm{F}_{1}=0.996$

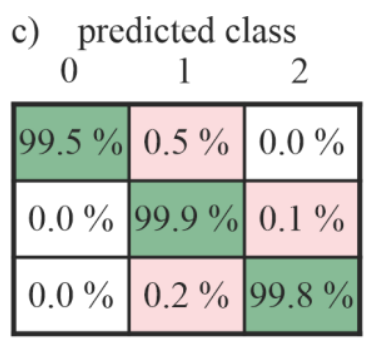

$\underline{F_{1}}=0.999$

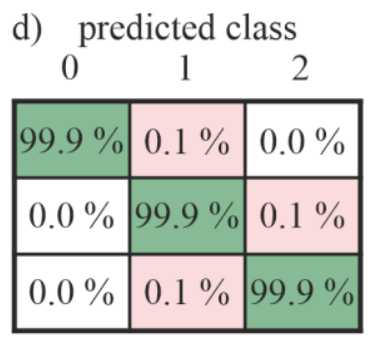

$\mathrm{F}_{1}=0.999$

$\mathrm{Han} / 98154 @ \mathrm{IFW}$

Fig. 3. Confusion matrices of teaching strategies for the process parameter sets with constant $a_{p}=1 \mathrm{~mm}$

In total, three of the four teaching strategies examined show an accuracy of more than $99 \%$ in the classification of materials. In general, the performance of material identification for process parameters constant $a_{p}$ increases with the number of trained process parameter sets. However, only two of the strategies discussed are suitable for achieving an $F_{1}$ score of over 0.999 . To cover a large process window with reduced training effort, the use of corner points of the process window for training is recommended for this application.

To evaluate the robustness of the NN with regard to geometric fluctuations, the range of the process parameter sets is extended by the third dimension by additionally considering the cutting depth. The feed and cutting speed used for this purpose vary between the process parameters considered so far. Based on this extended database, the ability of the NN to classify parameter sets with different $a_{p}$ using models created for a fixed $a_{p}$ is investigated. The change in the $a_{p}$, for example, is due to geometric deviations of the workpiece or processing strategies that provide a varying $a_{p}$ during the process. In addition, the increase in the classification rates is examined by including parameters with different cutting depths to the training set for the NN. The new process parameter sets and the teaching strategy are shown in Fig. 4.

Without new training parameters for the depth of cut, only the aircut is sufficiently detected, as depicted in Fig. 5a. While the test data with an $a_{p}$ of $1 \mathrm{~mm}$ were again classified with an $F_{1}$ score of 0.999 , the new test data could not be assigned robustly to the materials. Over all process parameters, only an $F_{1}$ score of 0.566 was reached. By adding process parameter sets with different $a_{p}$ to the training set, the classification performance could be improved, as depicted in Fig. 5b. With an $F_{1}$ score of 0.998 , almost all materials are correctly classified. As with the process parameter sets with constant $a_{p}$, different parameters must also be taken into account for varying $a_{p}$, if material classification on the basis of the NN should cover a wider range of $a_{p}$. 


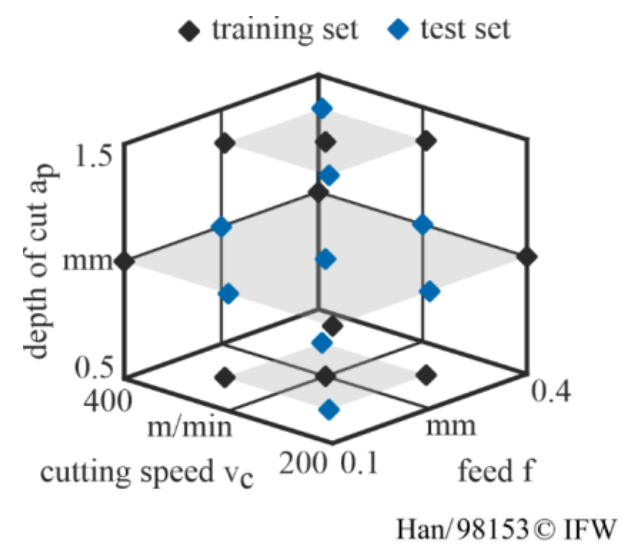

Fig. 4. Teaching strategy for parameter sets with changing $f, v_{c}$ and $a_{p}$

An NN is trained and tested with the depicted teaching strategy. For comparison, the NN based on the process parameters different $a_{p}$ is also tested. The results are shown in Fig. 5.
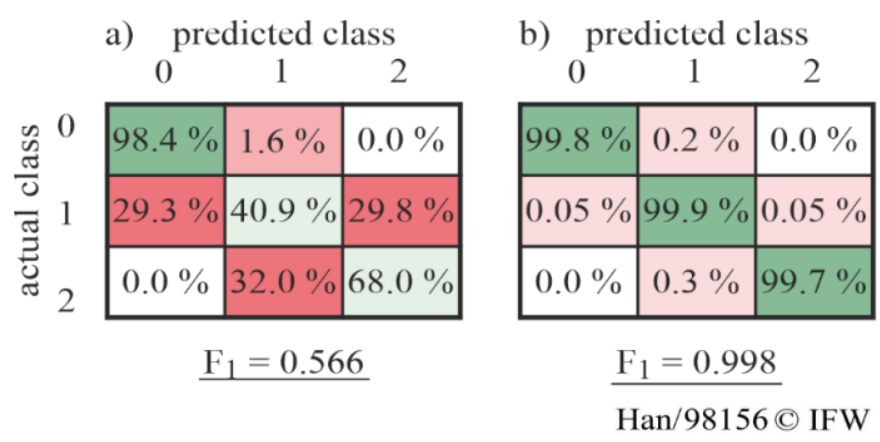

Fig. 5. Confusion matrices of the teaching strategies, a) without training $a_{p}$, b) with training $a_{p}$

From the investigations in this section, it can be concluded that for each varying parameter two or more different process parameter sets have to be considered to get a classification rate over $99 \%$. This applies in particular to process parameters which have a huge impact on the relevant features such as process forces or spindle torque. With this teaching strategy however, less than $60 \%$ of the process parameter sets have to be used for training the $\mathrm{NN}$, which results in a reduced amount of training data and a minimized training effort.

\section{IMPACT OF SIGNAL SOURCES ON THE RATE OF MATERIAL CLASSIFICATION WITH NN}

As discussed in the previous section, material identification for a process window with different cutting speed, feed and cutting depth is possible. So far, the ten best rated features have been used for training the NN. They were identified through the $S O F$ within the available feature set that is generated from measured signals of all signal sources. The measured signals 
can be assigned to three sources: machine signals from the NC, acceleration signals, and force signals that are measured with a dynamometer. Since the application of dynamometers is limited and the acquisition costs are high, dynamometers are rarely used in industrial process monitoring. To avoid the use of additional but not required sensors, signals, which have to be minimally available for material identification, are investigated.

Therefore, whether the force signals of the test bench can be neglected in NN training and which classification rate is achieved with the exclusive use of machine signals is investigated. For this purpose, the signal sources required for robust and sensitive material identification are investigated. Models with different feature sets are trained and the performance is compared.

Table 2. Ranked features for different signal source combinations: machine signals, acceleration and force

\begin{tabular}{|c|c|c|c|c|}
\hline Rank & All & Mach. \& Acc. & Machine signals & Acceleration \\
\hline 1 & $F_{c, \text { mean }}$ & $M_{\text {spindle,rms }}$ & $M_{\text {spindle,rms }}$ & $a_{z, \text { turret,rms }}$ \\
\hline 2 & $F_{c, \text { rms }}$ & $M_{\text {spindle,mean }}$ & $M_{\text {spindle,mean }}$ & $a_{z, \text { turret,std }}$ \\
\hline 3 & $M_{\text {spindle,rms }}$ & $I_{Z, \text { rms }}$ & $I_{Z, \text { rms }}$ & $a_{y, \text { turret,rms }}$ \\
\hline 4 & $M_{\text {spindle,mean }}$ & $I_{Z, \text { mean }}$ & $I_{Z, \text { mean }}$ & $a_{y, \text { turret,std }}$ \\
\hline 5 & $F_{f, \text { mean }}$ & $I_{X, \mathrm{rms}}$ & $I_{X, \text { rms }}$ & $a_{x, \text { turret,rms }}$ \\
\hline 6 & $F_{f, \text { rms }}$ & $I_{X, \text { mean }}$ & $I_{X, \text { mean }}$ & $a_{x, \text { turret,std }}$ \\
\hline 7 & $F_{p, \text { mean }}$ & $a_{z, \text { turret,rms }}$ & $I_{Z, \mathrm{p} 2 \mathrm{rms}}$ & $a_{x, \text { tool,rms }}$ \\
\hline 8 & $F_{p, \text { rms }}$ & $a_{z, \text { turret,std }}$ & $M_{\text {spindle,p2rms }}$ & $a_{x, \text { tool,std }}$ \\
\hline 9 & $I_{Z, \text { rms }}$ & $a_{y, \text { turret,rms }}$ & $I_{Y, \text { rms }}$ & $a_{z, \text { turret,p2p }}$ \\
\hline 10 & $I_{Z, \text { mean }}$ & $a_{y, \text { turret,std }}$ & $I_{Y, \text { mean }}$ & $a_{x, \text { turret,p2p }}$ \\
\hline
\end{tabular}

In total, four types of signal source combinations, presented in Table 2, are investigated. For each feature set, the ten best features of the signal source combination are selected. The signal source combination "all" is formed using all generated features. This configuration was

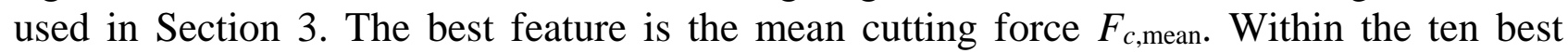
features, the force features appear six times compared to the machine signals that are represented four times. Moreover, no acceleration signal is listed within the ten best features. For the feature set "mach. \& acc.", only machine signals and accelerations were used. Here the spindle torque $M_{\text {spindle }}$ and the axis currents $I_{Y}$ and $I_{Z}$ as well as the accelerations of the turret $a_{y}$ and $a_{z}$ show the best SOF. If the force based signals are not considered, the spindle torque feature $\mathrm{M}_{\text {spindle,rms }}$ is the best feature. In the feature set "machine signals" and "acceleration", only machine signals and accelerations were used respectively. The SOF of the features based on the force signals and the spindle torque $M_{\text {spindle }}$ are the highest of all considered features. The features based on the axis currents $I_{Y}$ and $I_{Z}$ ranked second highest and the features of the acceleration signals came in last.

Based on these different feature sets, an NN was trained. The process parameter sets used for training and testing were the same for all feature sets and were identical to the configurations in Section 3. Figure 6 shows the resulting $F_{1}$ score of the different feature sets with constant and different $a_{p}$. For constant $a_{p}$, the teaching strategy from Fig. $2 \mathrm{c}$ was 
used, which considered varying cutting speed and feed. For different $a_{p}$, the teaching strategy from Fig. 4 was used, whereby the cutting depth was additionally taken into account.

In the case of a constant $a_{p}$, all features, except for the acceleration set, achieved an $F_{1}$ score over 0.99 . The $F_{1}$ score achieved with the acceleration feature set was only 0.696. This is because the acceleration signals, unlike the force-based or machine signals, have a high signal-to-noise ratio compared to the difference between the two materials. Therefore, the degree of separation between the first and second material is low and the identified material varies between two classes. Thus, it has been shown that for a classification rate above 0.99 , machine signals are sufficient and no additional sensors are needed.
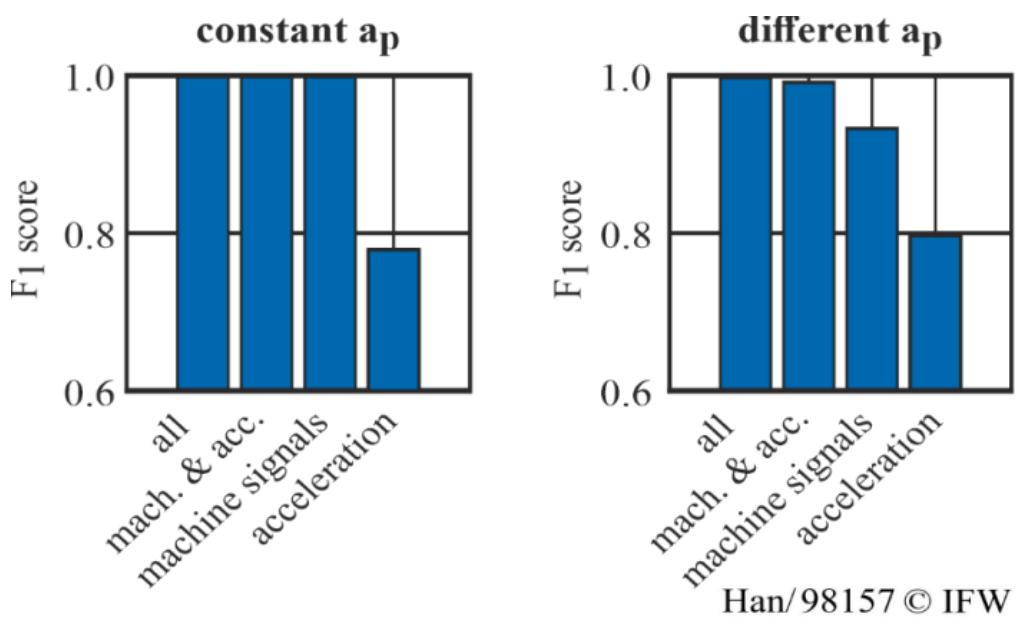

Fig. 6. $F_{1}$ score for different feature sets with constant and different $a_{p}$

In the case of different $a_{p}$, the feature set of the combination "all" had the best material classification with an $F_{1}$ score of 0.998 . The feature set with only acceleration signals showed the lowest classification rate. However, the classification rate significantly improved when features from acceleration signals were combined with features from machine signals. Thus, the NN trained with a combination of machine signal and acceleration features achieves a higher $F_{1}$ score than the NN trained with only one of these feature sources. Also, the $F_{1}$ score is only slightly lower than with an additional use of the force signals. To achieve a classification rate above 0.99 , it is sufficient to use a combination of features from machine signals and acceleration signals. Therefore, it is not necessary to use a dynamometer to measure the forces during the process.

It has been shown that an $F_{1}$ score of 0.99 can be achieved by using varying signal sources to train the NN. This implies a very high classification rate over all process parameter sets in the considered process window. However, whether the classification errors are evenly distributed over the entire test set or whether they only occur with certain process parameters is not taken into consideration. Therefore, the classification rate for the NN trained with machine signal and acceleration features is viewed separately for each of the nine process parameter sets. The predicted class for the entire test set is shown in Fig. 7b. As a reference, the measured cutting force, which was the best rated feature out of all features, and the acceleration of the turret in $\mathrm{z}$-direction is plotted in Fig. 7a. 
Material identification errors appear only at one process parameter set (test number 3). The material for all other process parameter sets is identified without errors. Therefore, it is not sufficient to only use the $F_{1}$ score to evaluate the classification rate of an NN. It is also necessary to investigate whether an accumulation of classification errors for certain process parameter sets exists. If this is the case, the neural network cannot be used for such process parameters.
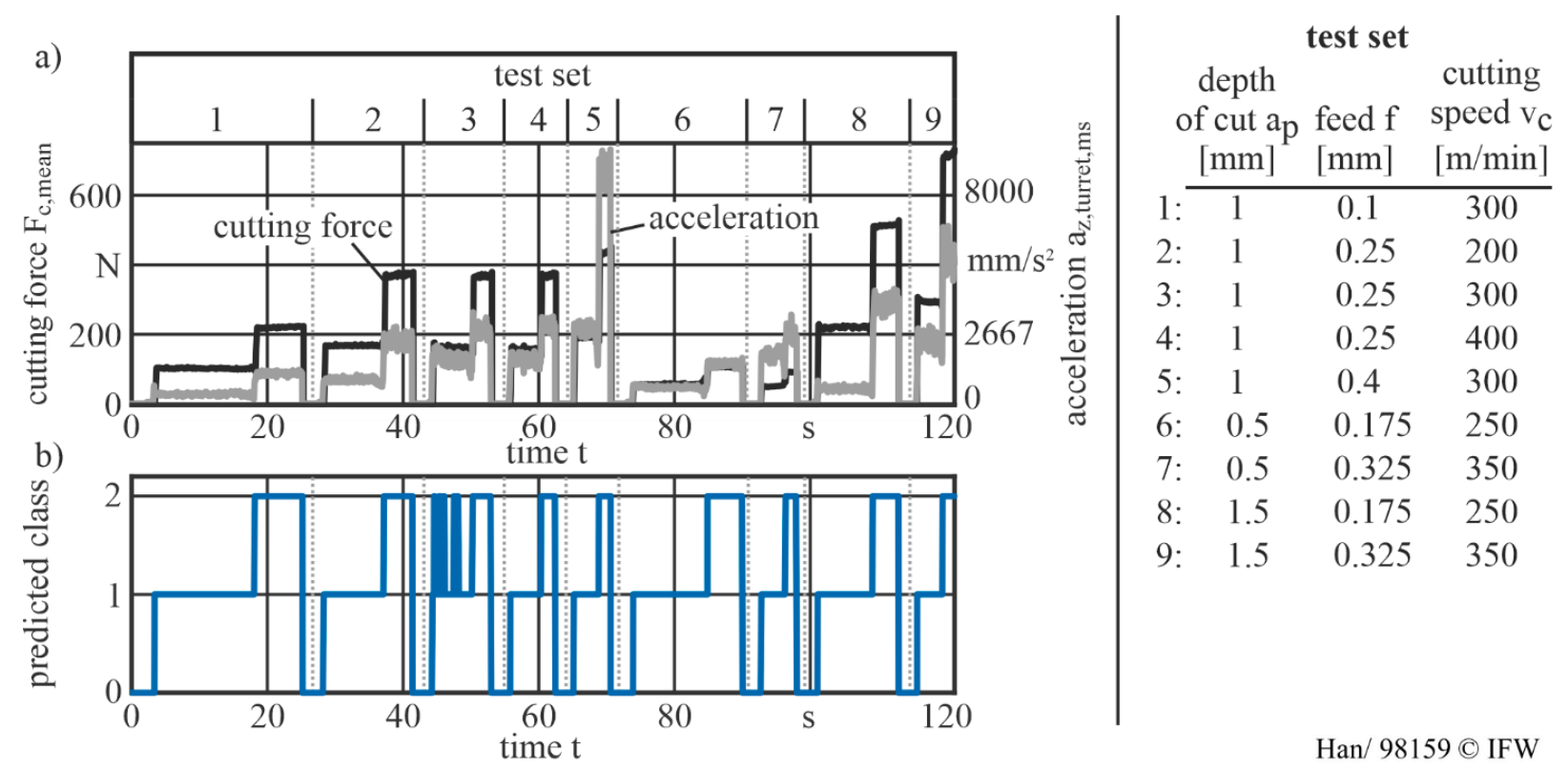

Fig. 7. Test of the NN with a feature set of signal source combinations "mach. \& acc."

In the present case, the material identification errors occurring at process parameter set number 3 are likely due to the cutting force amplitude of the aluminum overlapping with the cutting forces of the steel at a lower cutting depth for various process parameters. If the cutting depth is also taken into account during classification, this error does not occur anymore. However, this feature has not yet been included because the cutting depth is not provided by the NC and therefore additional knowledge is required.

\section{CONCLUSION}

Hybrid workpieces lead to new challenges for machining operations due to different material properties in a single workpiece. For this reason, it is necessary to identify the materials during the machining and to adapt the process parameters to the individual material. For the first time, this paper has shown that a neural network is suitable for classifying material for different feed, cutting speed and cutting depth during a turning process. If only machine signals are used for the classification of processes with constant cutting depth, a classification rate of $99.7 \%$ is achieved. For a normal distribution, this corresponds to a value of $\pm 3 \sigma$. This reference value is also used in process monitoring to generate envelopes and is therefore sufficiently accurate. This eliminates the need for 
additional external sensors. If the cutting depth is also varied, the parameter sets must also be included in the training process. The classification rate can be increased from $93.2 \%$ to $98.9 \%$ by using an additional acceleration sensor in combination with the machine control signals. An approach to increase this classification rate can be achieved by adding additional features such as information about the current depth of cut. The work contributes to increase the flexibility in material monitoring during the machining of hybrid components and to reduce the effort in training the NN.

In future work, the classification rate of unsupervised learning methods will be investigated for material separation to reduce the effort of preparing the training data. This will be compared with previous results, and further material compounds will be analyzed within the Collaborative Research Centre 1153.

\section{ACKNOWLEDGEMENTS}

The results presented in this paper were obtained within the Collaborative Research Centre 1153 "Process chain to produce hybrid high performance components by Tailored Forming' 'in the subproject B5 (project number: 252662854). The authors would like to thank the German Research Foundation (DFG) for the financial and organizational support of this project.

\section{REFERENCES}

[1] GOEDE M., STEHLIN M., RAFFLENBEUl L., KOPP G., BEEH E., 2009, Super Light Car-Lightweight Construction Thanks to a Multi-Material Design and Function Integration, European Transport Research Review, $1 / 1,5-10$.

[2] BEHRENS B.-A., BOUGUECHA A., FRISCHKORN C., HUSKIC A., STAKHIEVA.A., DURAN D., 2016, Tailored Forming Technology for Three Dimensional Components: Approaches to Heating and Forming, 5th Conference on Thermomechanical Processing, Milan, Italy.

[3] BEHRENS B.-A., BOUGUECHA A., VUCETIC M., PESHEKHODOV I., MATTHIAS T., KOLBASNIKOV N., SOKOLOV S., GANIN S., 2016, Experimental Investigations on The State of the Friction-Welded Joint Zone in Steel Hybrid Components After Process-Relevant Thermo-Mechanical, AIP Conf. Proc., 1769.

[4] BLOHM T., MILDEBRATH M., STONIS M., LANGNER J., HASSEL T., BEHRENS B.-A., 2017, Investigation of the Coating Thickness of Plasma-Transferred Arc Deposition Welded and Cross Wedge Rolled Hybrid Part, Production Engineering Research and Development, 11/3, 244-263.

[5] GOLDSTEIN R., BEHRENS B-A., DURAN D., 2017, Lightweighting by Tailored Forming: Bi-Material Stepped Shaft, Heat Treat 2017, Proceedings of the 29th ASM Heat Treating Society Conference, October 24-26, Columbus, Ohio, USA.

[6] BOEHNKE D., 2007, Qualitätsorientierte Zerspanung von Parallelverbunden im kontinuierlichen Schnitt, Dissertation, Leibniz Universität Hannover.

[7] OZSVÁTH P., SZMEJKÁL A., TAKÁCS J., EIDENHAMMER M., OBERMAIR F., 2006, Development of Face Milling Process for Mg-Hybrid (Mg-Al, Mg-Sintered Steel) Materials, Proceedings of the 7th Int. Conference on Magnesium Alloys and Their Applications, Wiley-VCH, 894-900.

[8] DENKENA B., BERGMANN B., BREIDENSTEIN B., PRASANTHAN V., WITT M., 2018, Analysis of Potentials to Improve the Machining of Hybrid Workpieces, Production Engineering Research and Development, $13 / 1,11-19$.

[9] GE Z., SONG Z., DING S., HUANG B., 2017, Data Mining and Analytics in the Process Industry: The Role of Machine Learning, IEEE Access, 5, 20590-20616.

[10] WUEST T., WEIMER D., IRGENS C., THOBEN K., 2016, Machine Learning in Manufacturing: Advantages, Challenges, and Applications, Production \& Manufacturing Research, 4/1, 23-45. 
[11] KILUNDU B., DEHOMBREUX P., CHIEMENTIN X., 2011, Tool Wear Monitoring by Machine Learning Techniques and Singular Spectrum Analysis, Mechanical Systems and Signal Processing, 25, 400-415.

[12] LAMRAOUI M., BARAKAT M., THOMAS M., BADAOUI M.E., 2013, Chatter Detection in Milling Machines by Neural Network Classification and Feature Selection, J. Vib. Control, 21, 1251-1266.

[13] DENKENA B., BERGMANN B., WITT M., 2018, Material Identification Based on Machine Learning Algorithms for Hybrid Workpieces During Cylindrical Operations, Journal of Intelligent Manufacturing, 30/6, $2449-2456$. 